\title{
Novel polar-coded space-time transmit diversity scheme over Rician fading MIMO channels
}

\author{
Bowen Feng, Shushi Gu, Jian Jiao* 토 , Shaohua Wu and Qinyu Zhang
}

\begin{abstract}
In this paper, a polar-coded space-time transmit diversity (STTD) scheme is proposed in order to improve the performance of multiple-input multiple-output (MIMO) system. In Rician fading MIMO channels, the corresponding polar-coded STTD system can be equivalent to a single transmission channel for each polar code bit. Density evolutions for the polar-coded STTD systems are proposed based on the analyses of the single transmission channel. The proposed density evolutions provide preferable guidance to construct polar codes for the polar-coded STTD systems. Simulation results show that the BER performance of polar-coded STTD system is significantly improved as the number of antennas increases and the influence of the variable Rician $K$-factor of MIMO channels gradually vanishes. The proposed $2 \times 2$ polar-coded STTD system can provide better frame error rate (FER) performance than low-density parity-check (LDPC)-based system under low and mid code rates, and the average advantage is $0.3 \mathrm{~dB}$ approximately when the code rate is 0.25 and $0.2 \mathrm{~dB}$ when the code rate is 0.5 .
\end{abstract}

Keywords: Polar code, Space-time transmit diversity, Density evolution, Rician fading

\section{Introduction}

Polar code introduced by Arıan can achieve the capacity of the memoryless symmetric channel with low encoding and decoding complexity [1]. A successive-cancellation (SC) decoding specialized for polar codes is proposed at the same time with the complexity $O(N \log N)$, which can provide acceptable bit error rate (BER) performance. The belief propagation (BP) decoding for polar codes is discussed in [2], and a modified BP decoder is given in order to improve the performance of polar codes for short lengths. The performance of BP decoder can also be improved by adapting the parity-check matrix [3]. Polar codes with cyclic redundancy check-aided (CRC-aided) successive-cancellation list (SCL) decoding [4] can provide the optimal BER performance, and the complexity is reduced in [5]. Polar codes with CRC-aided list decoding can outperform state-of-the-art low-density parity-check (LDPC) codes at short block lengths [6]. Thus, polar code, the highly efficient and reliable channel code, has many potential applications in wireless communications.
Multiple-input multiple-output (MIMO) systems have been widely applied in modern communication systems and will be still applied as a key technology for the nextgeneration wireless system with the number of antennas going large [7]. Diversity is an efficient technique to combat multipath fading in MIMO channels. The effect of transmit and receive diversity can be enhanced as the number of antennas increases $[8,9]$. Space-time transmit diversity (STTD) can achieve a full diversity gain with a simple structure, which can be regarded as a process of coding. The basic space-time coding (STC) model for two transmit antennas is Alamouti's scheme [10]. To improve the performance, channel coding is adopted in MIMO systems. STTD has been successfully combined with LDPC codes [11, 12] and Turbo codes [13], which enhanced the error-correcting performance but also induced huge encoding and decoding complexity. The complexity is mainly caused by the cost of LDPC and Turbo encoding and decoding algorithms. Thus, the low-complexity polar code can be a better choice to combine with STTD in MIMO systems. 
Density evolution (DE) is an important analytical tool for graph codes with message-passing decoding algorithms. The DE for polar codes based on the message passing in SC decoding graph is proposed in [14], which provides a preferable guidance to construct polar codes for obtaining better performance. In the process of the code construction, the selection of an information set is a key factor that impacts the performance of polar codes. Imprecise selection of the information set will aggravate the performance. The DE for polar codes provides a way to select the information set precisely for any discrete memoryless channel (DMC). An efficient way to construct polar codes based on DE is proposed in [15], which reduces the calculation cost.

In this paper, we extend the previous works on Rayleigh fading MIMO channels [16]. We analyze polar codes and STTD system in Rician fading MIMO channels of which the transmitter has known channel distribution information (CDI) and the receiver has channel side information (CSI). Some analyses and simulations about Rician fading channels and $K$-factors are given in [17]. In this paper, we consider the flat Rician fading channel model which is a suitable model for the fluctuations of the signal envelope in those narrowband multipath fading channels where there is a direct line-of-sight (LOS) path between the transmitter and the receiver [18]. The main contributions of this paper are stated as follows. Firstly, the polar-coded STTD system model is proposed, which provides the guidance on how to concatenate the polar coding and STTD. With the analyses, we convert the proposed system to an equivalent single fading transmission channel for each polar code bit. It can help construct suitable polar codes for the proposed system and improve the performance. Secondly, the parameters of the equivalent fading channel are calculated based on the Rician distribution. The DE for the polar-coded STTD system is proposed based on the parameters. We describe the DEs for the systems with different numbers of antennas in this paper. Furthermore, the proposed DE guides the construction of polar codes in the systems. We describe how to construct suitable polar codes for proposed system based on the DEs. At last, simulation results are provided for the BER performance of polar-coded STTD systems in Rician MIMO channels with variable Rician $K$-factors. Simulation results of frame error rate (FER) performance are also provided with different code rates. This paper includes a performance comparison of the proposed polar-coded STTD system and the state-of-the-art LDPC-based system.

The remainder of the paper is organized as follows. Section 2 describes the preliminaries of polar codes and space-time coding. Section 3 proposes the model of polarcoded STTD system. DEs for polar-coded STTD systems are also proposed. Simulation results of the polar-coded
STTD systems are provided in Section 4. Finally, Section 5 concludes the paper.

\section{Preliminaries}

\subsection{Successive-cancellation (SC) decoding}

The SC decoding specialized for polar codes is proposed in [1] with the complexity $O(N \log N)$. The $\mathrm{SC}$ decoder outputs bit by bit estimation of the sources. When we need to decode the $i$ th source bit $u_{i}$, the prior bits $u_{1}^{i-1}$ which have been decoded are regarded as known constants and substituted into the decoding calculation. In SC decoding calculations, the log-likelihood ratio (LLR) for the $i$ th bit is defined as

$$
L_{N}^{(i)}\left(y_{1}^{N}, \hat{u}_{1}^{(i-1)}\right)=\ln \frac{W_{N}^{(i)}\left(y_{1}^{N}, \hat{u}_{1}^{i-1} \mid 0\right)}{W_{N}^{(i)}\left(y_{1}^{N}, \hat{u}_{1}^{i-1} \mid 1\right)},
$$

where $u_{1}^{i-1}=\left(u_{1}, \ldots, u_{i-1}\right)$ denotes an $(i-1)$ dimensional source vector and $y_{1}^{N}=\left(y_{1}, \ldots, y_{N}\right)$ denotes the output of the channels. The LLR for each bit can be calculated based on the recursive formulas as follows,

$$
\begin{aligned}
& L_{N}^{(2 i-1)}\left(y_{1}^{N}, \hat{u}_{1}^{2 i-1}\right) \\
& =2 \tanh ^{-1}\left(\tanh \left(L_{N / 2}^{(i)}\left(y_{1}^{N / 2}, \hat{u}_{1, o}^{2 i-2} \oplus \hat{u}_{1, e}^{2 i-2}\right) / 2\right)\right. \\
& \left.\quad \times \tanh \left(L_{N / 2}^{(i)}\left(y_{N / 2+1}^{N}, \hat{u}_{1, e}^{2 i-2}\right) / 2\right)\right), \\
& L_{N}^{(2 i)}\left(y_{1}^{N}, \hat{u}_{1}^{2 i-1}\right)=L_{N / 2}^{(i)}\left(y_{N / 2+1}^{N}, \hat{u}_{1, e}^{2 i-2}\right) \\
& +(-1)^{\hat{u}_{2 i-1}} L_{N / 2}^{(i)}\left(y_{1}^{N / 2}, \hat{u}_{1, o}^{2 i-2} \oplus \hat{u}_{1, e}^{2 i-2}\right),
\end{aligned}
$$

where $\hat{u}_{1, o}^{i}$ and $\hat{u}_{1, e}^{i}$ denote the subvectors consisting of elements of $\hat{u}_{1}^{i}$ with odd and even indices and $\oplus$ denotes modulo- 2 addition. Then, the $i$ th bit can be determined as 0 or 1 after obtaining the corresponding LLR.

The SCL decoding algorithm is proposed in [4] as an upgrade version of the SC. SCL can be regarded as a joint SC algorithm and maximum likelihood (ML) algorithm. In the process of SCL decoding, source bit is not decoded immediately at each step. Instead, a maximum of $l$ candidate paths are considered. For each source bit $u_{i}$, SCL doubles the number of decoding paths by pursuing both $u_{i}=0$ and $u_{i}=1$ options and then preserves the most likely $l$ paths in a list and discards others. When all source bits are traversed, the most reliable path is chosen as the output of decoder. The complexity of SCL decoding is $O(l N \log N)$. SCL decoding is often combined with CRC for improving the performance of decoding. The CRC-aided SCL decoding provides better FER performance than other decoding methods according to [19]. An LLR-based SCL decoding provided in [20] 
is a numerically stable implementation of SCL decoding, which can be formulated in the LLR domain.

In [14], each step of SC is regarded as a BP decoding, where a decoding process of $i$ th bit is regarded as a depth$n$ tree. Furthermore, the decoding tree is characterized by the binary expansion $b_{n} \cdots b_{1}$ of $(i-1)$, such that nodes at depth- $t$ of the decoding tree are check nodes and variable nodes if $b_{t}=0$ and $b_{t}=1$, respectively. As shown in Fig. 1, the decoding tree for 4th bit is shown by thick solid lines when $n=3$. Known edges are shown by dashed lines. Edges which are not used for decoding of the $i$ th bit are shown by thin solid lines. The binary expansion of $(i-1)$ is 011 . The nodes at depth-1 and depth-2 correspond to variable nodes, and the nodes at depth- 3 are check nodes. LLRs are recursively evaluated in the tree based on the formulas (2) and (3) at check and variable nodes, respectively. Thus, density evolution, an analytical tool for graph codes, can be adopted for polar codes based on the LLRs.

\subsection{Density evolution for polar codes}

Polar codes are generated by source bits including variable information bits and fixed frozen bits. The indices of information bits form an information set. The selection of the information set is a key factor that impacts the performance of polar codes. The process of channel polarization provided in [1] shows the basis of the information set selection. The polarized channel $W_{N}$ combined by $N$ DMCs $W$ is split to parallel subchannels $\left\{W_{N}^{(i)}\right\}$ whose capacities are different. DE for polar codes guides the selection of the information set by ranking the probability of incorrect messages of subchannels. The indices of the subchannels with lowest probabilities of the incorrect messages are selected as the information set. The probability of incorrect messages can be obtained by calculating the probability distribution functions (PDFs) of LLRs passing in the decoding graph, where the PDFs are regarded as the densities. For example, the LLR of the $i$ th subchannel $W_{N}^{(i)}$ is regarded as a variable, then the density of the subchannel can be expressed as $\mathbf{a}_{N}^{(i)}(z)$ that is the PDF of the variable. When all zero bits are transmitted and the channel $W$ is symmetric, the probability of incorrect messages of the $i$ th subchannel can be expressed as $P_{e}(i)=\int_{-\infty}^{0} \mathbf{a}_{N}^{(i)}(z) \mathrm{d} z$. The densities passing in the SC decoding graph can be calculated as follows,

$$
\mathbf{a}_{2 N}^{(2 i)}=\mathbf{a}_{N}^{(i)} \star \mathbf{a}_{N}^{(i)}, \mathbf{a}_{2 N}^{(2 i-1)}=\mathbf{a}_{N}^{(i)} \oslash \mathbf{a}_{N}^{(i)}, \mathbf{a}_{1}^{(1)}=\mathbf{a}_{W}
$$

where $\mathbf{a}_{W}$ is the PDF of the initial channel W's LLR when 0 is transmitted and where $\star$ and $\square$ are the convolution operations for variable nodes and check nodes, respectively [21]. Based on the densities of all subchannels, the corresponding probabilities of incorrect messages can be calculated. With ranking of the probabilities of incorrect messages, the information set is determined.

\subsection{Space-time coding}

Space-time coding is a simple coding technique to achieve transmit diversity. The basic STC model, Alamouti's scheme proposed for $2 \times 1$ multiple-input and singleoutput (MISO) channel and $2 \times 2$ MIMO channel, has simple encoding and decoding operations. The encoding matrix of Alamouti's scheme is

$$
\mathbf{G}_{2}=\left[\begin{array}{ll}
x_{1} & -x_{2}^{*} \\
x_{2} & x_{1}^{*}
\end{array}\right]
$$

where the source bits $x_{1}$ and $x_{2}$ in the first column are separately transmitted by the two antennas in the first transmission period and the complex conjugations of them in the second column are separately transmitted in the second transmission period. We assume that each point-to-point channel in MIMO follows a Rician fading model, where the channel gains are constant across two

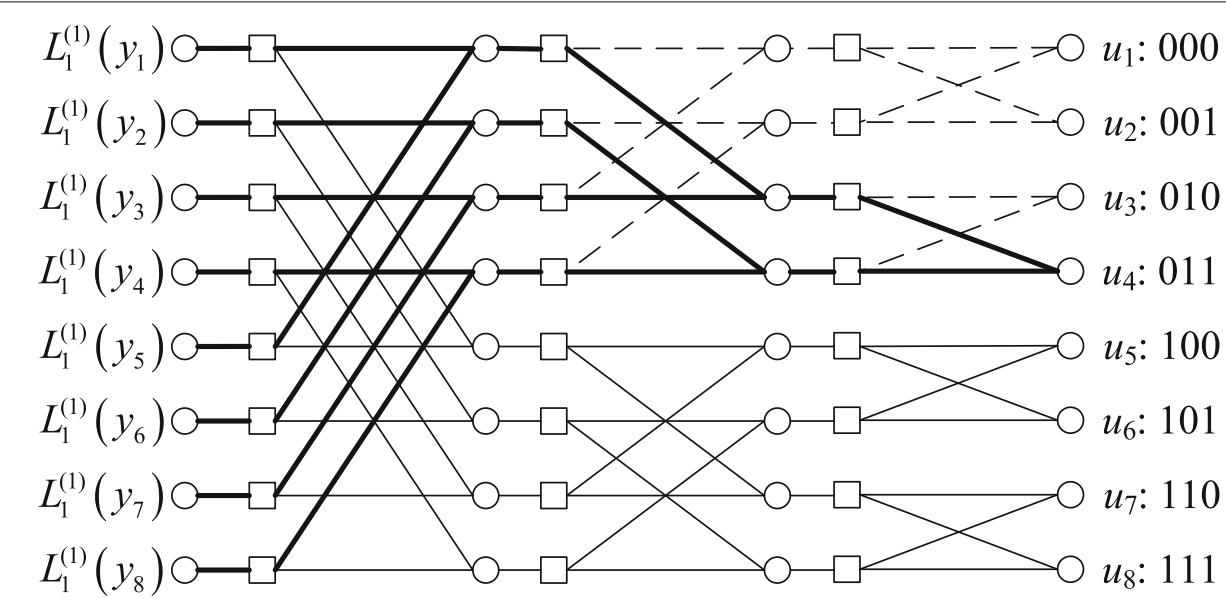

Fig. 1 Decoding graph of polar codes, $N=8$. The recursive processes of the $S C$ decoding for polar codes with the length $N=8$. The thick solid lines show the decoding tree for the 4 th bit 
consecutive transmission periods. The signals received by the $j$ th antenna in two periods can be expressed as

$$
\begin{aligned}
& r_{j 1}=h_{1 j} x_{1}+h_{2 j} x_{2}+n_{j 1}, \\
& r_{j 2}=-h_{1 j} x_{2}^{*}+h_{2 j} x_{1}^{*}+n_{j 2},
\end{aligned}
$$

where $h_{i j}$ is the channel gain between the $i$ th transmit antenna and the $j$ th receive antenna following a Rician distribution and where $n_{j t}$ is an additive Gaussian noise with zero mean and variance $\sigma_{n}^{2}$ at the $j$ th receive antenna in the $t$ th period. Decoding of STC is a two-step process, combining and maximum likelihood decoding. We assume that there is only one receive antenna. Combiner combines the received signals based on the estimation of CSI as follows

$\tilde{x}_{1}=h_{11}^{*} r_{11}+h_{21} r_{12}^{*}=\left(\left|h_{11}\right|^{2}+\left|h_{21}\right|^{2}\right) x_{1}+h_{11}^{*} n_{11}+h_{21} n_{12}^{*}$, $\tilde{x}_{2}=h_{21}^{*} r_{11}-h_{11} r_{12}^{*}=\left(\left|h_{11}\right|^{2}+\left|h_{21}\right|^{2}\right) x_{2}-h_{11} n_{12}^{*}+h_{21}^{*} n_{11}$,

where $\tilde{x}_{1}$ and $\tilde{x}_{2}$ are the outputs of the combiner, and the distributions of them are same obviously, and where source bits $x_{1}$ and $x_{2}$ are isolated from the received signals. Then, the combined signals are sent to maximum likelihood decoder which can give out the source bits $x_{1}$ and $x_{2}$. The generalized space-time code model proposed for more antennas can be designed based on orthogonal theories.

\section{Density evolution method for polar-coded STTD system}

In this section, we will propose a polar-coded STTD system model in which the estimation of channel information is considered. We assume that the CDI of Rician MIMO channel is known at the transmitter and perfect estimation of CSI is known at the receiver. DEs will be also proposed for polar-coded STTD systems with different numbers of antennas.

\subsection{Model of polar-coded STTD system}

In Rician fading MIMO channel, the model of each point-to-point channel is comprised of a multiplicative gain coefficient and an additive noise. The gain coefficient follows the Rician distribution $h_{i j} \sim \frac{h_{i j}}{\sigma_{h}^{2}} \exp (-$ $\left.\frac{h_{i j}^{2}+\beta^{2}}{2 \sigma_{h}^{2}}\right) I_{0}\left(\frac{h_{i j} \beta}{\sigma_{h}^{2}}\right)$, where $I_{0}(\cdot)$ is the zero-order modified Bessel function of the first kind. $\beta$ is the amplitude of the specular signal component which can be calculated from the Rician $K$-factor as $\beta=\sqrt{2 \sigma_{h}^{2} K}$, in which $K$ is a pure number. The Rician $K$-factor is often expressed in decibels $(\mathrm{dB})$ in practice, which will be applied in the simulations in this paper. The additive noise of a point-to-point channel model follows the Gaussian distribution $\mathcal{N}\left(0, \sigma_{n}^{2}\right)$.

As shown in Fig. 2, a polar-coded STTD system model is proposed. It can be regarded as a kind of concatenated coding, where the polar codes are the outer codes and the STC codes are inner codes. The outer polar codes are generated based on transmitter's CDI, then sent to the STC encoder. The output concatenated codes after passing through the Rician fading MIMO channels are obtained by the receive antennas. The received signals are combined by the STC combiner, then decoded by the polar code decoder. The SC, SCL, and other decoders for polar codes can be adopted in the system.

\subsection{Density evolution for polar-coded STTD system with two transmit antennas and one receive antenna}

Now, we focus on the DE for polar-coded STTD system to guide the construction of polar codes in the system. We assume that there are two transmit antennas and one receive antenna in the proposed polar-coded STTD system. Alamouti's scheme is adopted to construct STC. In the system, the output of the STC combiner can be expressed as,

$$
y_{i}=\left(\left|h_{11}\right|^{2}+\left|h_{21}\right|^{2}\right) x_{i}+h_{11}^{*} n_{11}+h_{21} n_{12}^{*},
$$

where $x_{i}$ is any modulated polar code bit. Each combined signal $y_{i}$ can be regarded as an output of a fading channel where a polar code bit $x_{i}$ traverses. Thus, the polar-coded STTD system can be equivalent to a single fading transmission channel for each polar code bit. The gain coefficient of the equivalent channel can be expressed as $\sum_{q}\left|h_{q}\right|^{2}$. The i.i.d variable $\left|h_{q}\right|^{2}$ can be regarded as a square sum of two nonzero mean Gaussian variables, so the variable $\sum_{q}\left|h_{q}\right|^{2}$ follows a noncentral chi-square distribution

$$
f_{h}\left(h=\sum_{q=1}^{n}\left|h_{q}\right|^{2}\right)=\frac{h^{(n-1) / 2}}{2 \sigma_{h}^{2} \beta^{n-1}} \exp \left(-\frac{h+\beta^{2}}{2 \sigma_{h}^{2}}\right) I_{n-1}\left(\sqrt{\frac{\beta^{2} h}{\sigma_{h}^{4}}}\right),
$$

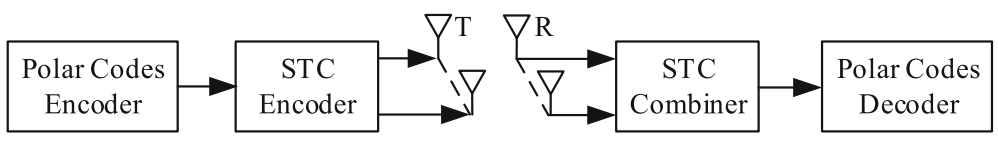

Fig. 2 Model of polar-coded STTD system. The running process of the concatenation of polar coding and STTD. It shows the overall delivery process of the information bits from the transmitter to the receiver 
where $I_{n-1}(\cdot)$ is the $n-1$-order modified Bessel function of the first kind. For $2 \times 1$ polar-coded STTD system, the gain coefficient of the equivalent channel is expressed as $h=\left|h_{11}\right|^{2}+\left|h_{21}\right|^{2}$. Now, each output $y_{i}$ can be regarded as a Gaussian random variable with mean $h$ and variance $h \sigma_{n}^{2}$. The conditional PDF of $y_{i}$ can be expressed as $p\left(y_{i} \mid x_{i}, h\right)=\frac{1}{\sqrt{2 \pi h \sigma_{n}^{2}}} \exp \left(-\frac{\left(y_{i}-h x\right)^{2}}{2 h \sigma_{n}^{2}}\right)$, and the initial LLR is obtained by

$$
L_{W}=\ln \frac{p\left(y_{i} \mid x_{i}=-1, h\right)}{p\left(y_{i} \mid x_{i}=1, h\right)}=-\frac{2 y_{i}}{\sigma_{n}^{2}} \quad .
$$

The initial LLR $L_{W}$ is a Gaussian variable with mean $2 h / \sigma_{n}^{2}$ and variance $4 h / \sigma_{n}^{2}$. Now, we can derive the PDF of the initial LLR by using the distribution of $h$. We assume that each $h_{i j}$ is a normalized Rician fading factor. Substituting $n=2$ to function (9), we can obtain the distribution of $h$. Finally, the PDF of the initial LLR can be expressed as

$\mathbf{a}_{W}(z)=\int_{0}^{\infty} \frac{\sigma_{n}}{\sqrt{8 \pi} \beta} \exp \left(-\frac{\left(z-\frac{2}{\sigma_{n}^{2}} h\right)^{2}}{8 h / \sigma_{n}^{2}}-h-\beta^{2}\right) I_{1}\left(\sqrt{4 \beta^{2} h}\right) \mathrm{d} h$.

Thus, densities of subchannels can be calculated by (4). Corresponding probabilities of incorrect messages can also be calculated. Then, the information set can be selected.

Now, we focus on the symmetry of the proposed DE, which is a key property when using DE in symmetric channels. The symmetry of DE is defined in [22]. If the density $\mathbf{a}_{W}(z)$ satisfies

$$
\mathbf{a}_{W}(z)=\mathbf{a}_{W}(-z) \exp (z),
$$

the density $\mathbf{a}_{W}(z)$ is symmetric. When substituting (11) to (12), we find that the equation is true and the initial density for $2 \times 1$ polar-coded STTD system is symmetric.

\subsection{Density evolution for polar-coded STTD system with} two transmit antennas and two receive antennas

We now analyze the polar-coded STTD system corresponding to Rician fading $2 \times 2$ MIMO channel. The output of the STC combiner can be expressed as

$$
\begin{aligned}
y_{i}= & \left(\left|h_{11}\right|^{2}+\left|h_{21}\right|^{2}+\left|h_{12}\right|^{2}+\left|h_{22}\right|^{2}\right) x_{i} \\
& +h_{11}^{*} n_{11}+h_{21} n_{12}^{*}+h_{12}^{*} n_{21}+h_{22} n_{22}^{*} .
\end{aligned}
$$

Now, the gain coefficient of the equivalent channel is expressed as $h=\left|h_{11}\right|^{2}+\left|h_{21}\right|^{2}+\left|h_{12}\right|^{2}+\left|h_{22}\right|^{2}$. Each output $y_{i}$ can still be regarded as a Gaussian random variable with mean $h$ and variance $h \sigma_{n}^{2}$. Substitute $n=4$ to function (9) to get the distribution of $h$. The PDF of the initial LLR is as follows $\mathbf{a} W(z)=\int_{0}^{\infty} \frac{\sigma_{n} h}{\sqrt{8 \pi} \beta^{3}} \exp \left(-\frac{\left(z-\frac{2}{\sigma_{n}^{2}} h\right)^{2}}{8 h / \sigma_{n}^{2}}-h-\beta^{2}\right) I_{3}\left(\sqrt{4 \beta^{2} h}\right) \mathrm{d} h$.

When substituting (14) to (12), we find that the initial density for $2 \times 2$ polar-coded STTD system is symmetric.

3.4 Density evolution for polar-coded STTD system with four transmit antennas and two receive antennas

The generate matrix of STC for four transmit antennas is given as follows,

$$
\mathbf{G}_{4}=\left[\begin{array}{llll}
x_{1} & x_{2} & x_{3} & x_{4}^{*} \\
-x_{2}^{*} & x_{1}^{*} & -x_{4} & x_{3}^{*} \\
-x_{3}^{*} & x_{4} & x_{1}^{*} & -x_{2}^{*} \\
-x_{4}^{*} & -x_{3} & x_{2} & x_{1}
\end{array}\right]
$$

We adopt this STC scheme in the $4 \times 2$ polar-coded STTD system. Now, the output of the combiner can be expressed as

$$
\begin{aligned}
y_{i}= & \left(\left|h_{11}\right|^{2}+\left|h_{21}\right|^{2}+\left|h_{31}\right|^{2}+\left|h_{41}\right|^{2}+\left|h_{12}\right|^{2}+\left|h_{22}\right|^{2}\right. \\
& \left.+\left|h_{32}\right|^{2}+\left|h_{42}\right|^{2}\right) x_{i}+h_{11}^{*} n_{11}+h_{21} n_{12}^{*}+h_{31} n_{13}^{*} \\
& +h_{41}^{*} n_{14}+h_{12}^{*} n_{21}+h_{22} n_{22}^{*}+h_{32} n_{23}^{*}+h_{42}^{*} n_{24} .
\end{aligned}
$$

The distributions of gain coefficient and additive noise for each output signal $y_{i}$ are same respectively. The gain coefficient of the equivalent channel is expressed as $h=\left|h_{11}\right|^{2}+\left|h_{21}\right|^{2}+\left|h_{31}\right|^{2}+\left|h_{41}\right|^{2}+\left|h_{12}\right|^{2}+\left|h_{22}\right|^{2}+\mid$ $\left.h_{32}\right|^{2}+\left|h_{42}\right|^{2}$. We can substitute $n=8$ to function (9). Now, the PDF of the initial LLR can be expressed as

$\mathbf{a}_{W}(z)=\int_{0}^{\infty} \frac{\sigma_{n} h^{3}}{\sqrt{8 \pi} \beta^{7}} \exp \left(-\frac{\left(z-\frac{2}{\sigma_{n}^{2}} h\right)^{2}}{8 h / \sigma_{n}^{2}}-h-\beta^{2}\right) I_{7}\left(\sqrt{4 \beta^{2} h}\right) \mathrm{d} h$.

This initial density for $4 \times 2$ polar-coded STTD system is also symmetric obviously.

\section{Results and simulations}

We have implemented the DEs for polar-coded STTD system. In this section, we will focus on the BER and FER performance of the proposed polar-coded STTD systems.

\subsection{BER performance of polar-coded STTD systems in Rician MIMO channels with different $K$-factors}

Simulations for the BER performance of the polar-coded STTD systems respectively corresponding to $2 \times 1,2 \times 2$, and $4 \times 2$ Rician fading MISO/MIMO channels are provided where the polar codes are constructed based on the proposed DEs. The length of the polar codes is 1024, and the rate is 0.5. SC decoding is applied in the BER simulations. Rician fading channels with different Rician 
$K$-factors are adopted in the simulations. We apply BPSK to modulate the signals.

Figure 3 shows the BER performance of polar-coded STTD system with two transmit antennas and one receive antenna corresponding to the $2 \times 1$ MISO. When the Rician $K$-factor in $\mathrm{dB}$ approaches positive infinity, the point-to-point Rician channel in MIMO can be regarded as a Gaussian channel. When it approaches negative infinity, the point-to-point Rician channel can be regarded as a Rayleigh channel. The BER performance of the $2 \times 1$ system can reach $10^{-4}$ when $K=0 \mathrm{~dB}$ and $E_{b} / N_{0}$ is $3.9 \mathrm{~dB}$ approximately. Here, we consider that the BER $10^{-4}$ and FER $10^{-3}$ are sufficient to meet the actual demand for common scenarios. The similar performance indicators have been also adopted in many relevant works, such as $[5,23,24]$ and [25]. As Rician $K$-factor increases, the BER performance of the system is significantly improved. For example, there is an approximate $0.2-\mathrm{dB}$ improvement on BER performance of the $2 \times 1$ system as $K$-factor increases from 0 to $3 \mathrm{~dB}$.

As the number of transmit and receive antennas increases, the BER performance is significantly improved. As shown in Fig. 4, the BER performance of the $2 \times 2$ system can reach $10^{-4}$ when $K=0 \mathrm{~dB}$ and $E_{b} / N_{0}$ is $0.6 \mathrm{~dB}$ approximately, which obtains an approximate 3.3$\mathrm{dB}$ improvement over the $2 \times 1$ system. This performance is further improved by $3.2 \mathrm{~dB}$ by the $4 \times 2$ polar-coded
STTD system as shown in Fig. 5. On the other hand, as the number of antennas increases, STC becomes a more dominant influence factor on BER performance than variable Rician $K$-factor. Figure 4 shows that there is only an approximate $0.1-\mathrm{dB}$ improvement on BER performance of the $2 \times 2$ system as $K$-factor increases from 0 to $3 \mathrm{~dB}$. Moreover, this improvement is further reduced in the $4 \times 2$ polar-coded STTD system as shown in Fig. 5 . The Rician $K$-factor has less and less influence on BER performance as antenna structure in the system enlarges. The performance difference of the $2 \times 1$ system is about two orders of magnitude between Rayleigh fading and AWGN channel models. However, for $4 \times 2$ polar-coded STTD system, the differences of performance with variable $K$ factors and fixed $E_{b} / N_{0}$ are only in an order of magnitude approximately.

\subsection{FER performance of polar-coded STTD system with different code rates}

According to the simulations above, we acknowledge that the performance of $2 \times 2$ polar-coded STTD system is sufficient for actual needs, and the complexity of it is low relatively. Thus, we focus on the FER performance of the proposed $2 \times 2$ polar-coded STTD system now. Firstly, we test the FER performance of the polar-coded STTD system and compare it with the FER performance of the LDPC-based STTD system [11]. In the simulations,

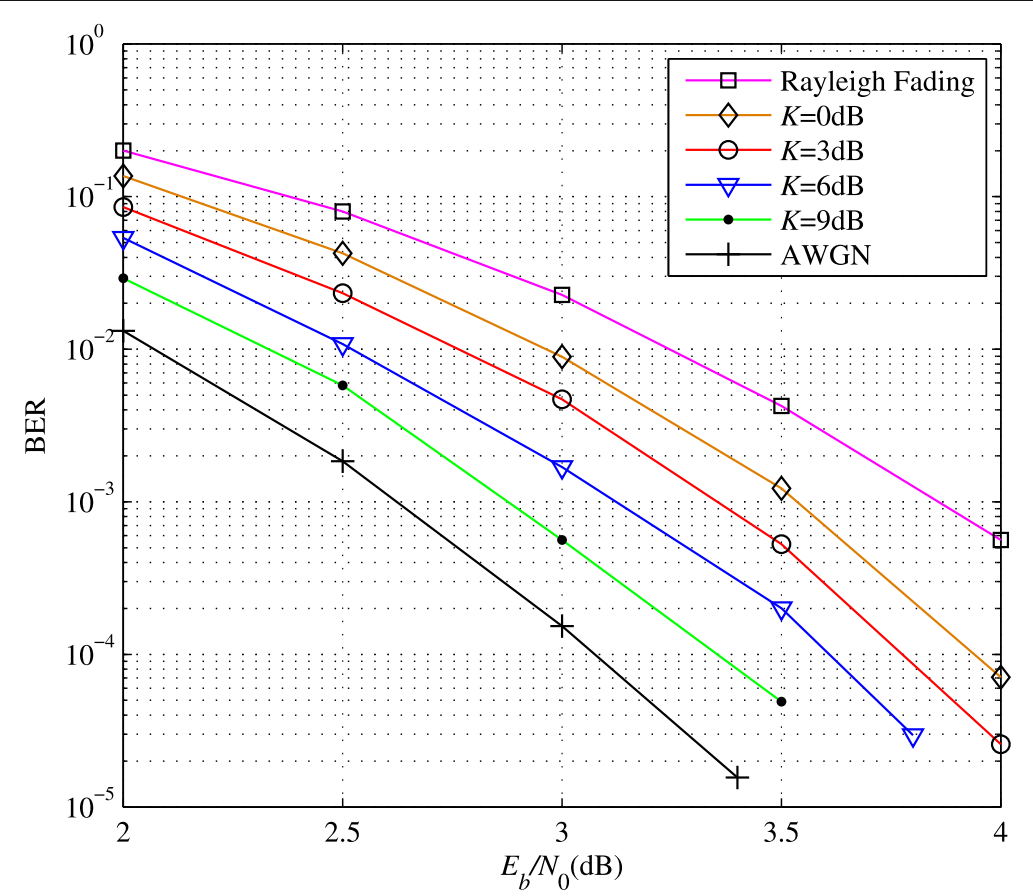

Fig. 3 BER performance of polar-coded STTD system corresponding to $2 \times 1$ MISO. The bit error rate performance of polar-coded STTD system with two transmit antennas and one receive antenna. The Rician $K$-factors are various, which include the positive infinity (AWGN) and negative infinity (Rayleigh) 


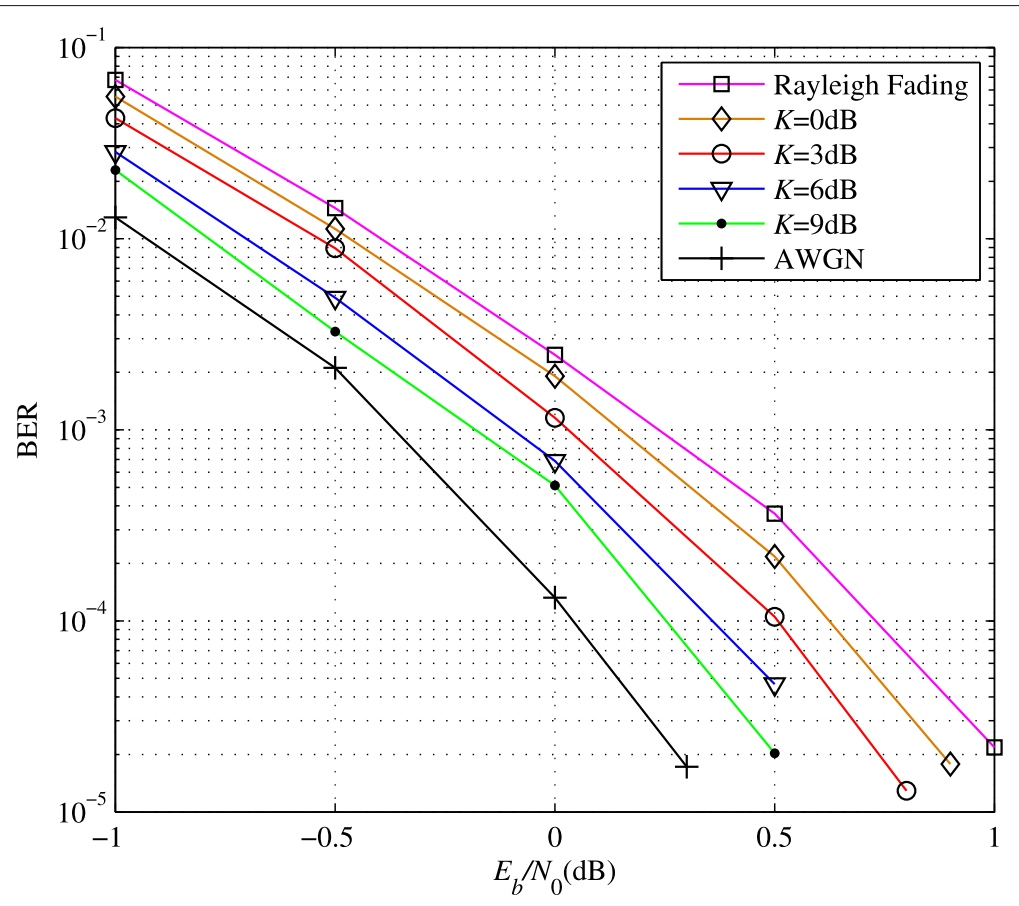

Fig. 4 BER performance of polar-coded STTD system corresponding to $2 \times 2 \mathrm{MIMO}$. The bit error rate performance of polar-coded STTD system with two transmit antennas and two receive antennas. The Rician $K$-factors are various, which include the positive infinity (AWGN) and negative infinity (Rayleigh)

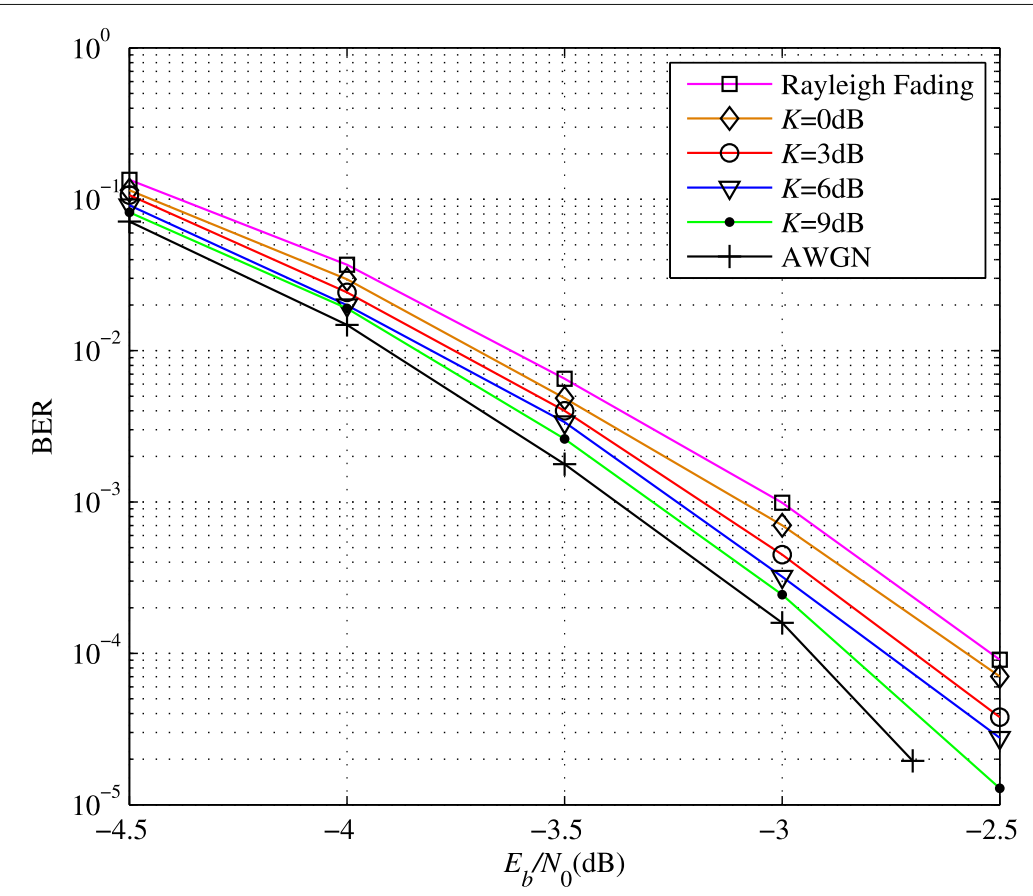

Fig. 5 BER performance of polar-coded STTD system corresponding to $4 \times 2$ MIMO. The bit error rate performance of polar-coded STTD system with four transmit antennas and two receive antennas. The Rician $K$-factors are various, which include the positive infinity (AWGN) and negative infinity (Rayleigh) 
the block length of the outer polar codes or LDPC codes is 1024 , and the code rates are $0.25,0.5$, and 0.75 . The Rician $K$-factor of the channel is assumed as $0 \mathrm{~dB}$. The $\mathrm{SC}$ decoding for the polar codes is still adopted in the simulation. In addition, the CRC-aided SCL decoding is adopted in order to show the optimal performance of the system. Twenty-four CRC parity bits are attached to every input frame. The list size of CRC-aided SCL is $l=32$. At this point, the complexity of the SCL decoding is as $O(l N \log N)=O(327680)$. In the simulations, the regular LDPC codes [26] are adopted in the LDPC-based STTD system with the average of variable degree distribution $\bar{d}_{v}=4$. We set the maximum BP iteration as $I_{\max }=40$ in order to balance the complexity of the BP decoding for LDPC codes with the one of the SCL decoding for polar codes. At this point, the complexity of the BP decoding is as $O\left(I_{\max }\left(2 N \bar{d}_{v}\right)\right)=O(327680)$.

As shown in Fig. 6, CRC-aided SCL decoding improves the FER performance of polar-coded STTD system significantly. It obtains a 0.3-dB improvement than SC decoding approximately for each simulated code rate. When the code rate is $R=0.25$, polar-coded STTD system with the help of CRC-aided SCL decoding provides an average $0.3 \mathrm{~dB}$ advantage over LDPC-based STTD system in FER. When the code rate increases to $R=0.5$, LDPC-based STTD system provides better FER performance than polar-coded STTD system with SC decoding under high $E_{b} / N_{0}$. However, it cannot exceed polar-coded STTD system with CRC-aided SCL decoding, and the FER performance gap is $0.2 \mathrm{~dB}$ approximately. When the code rate reaches $R=0.75$, LDPC-based STTD system shows advantages under high code rate. Its FER performance is slightly better than that of polar-coded STTD system with CRC-aided SCL decoding. Above all, CRCaided SCL decoding can help polar-coded STTD system provide optimal FER performance under low and mid code rates.

Moreover, we make an FER performance comparison of the proposed polar-coded STTD system and the state-ofthe-art LDPC-based system, in which the state-of-the-art LDPC codes provided in Verizon's document [27] are applied. In the simulations, the block length of the outer

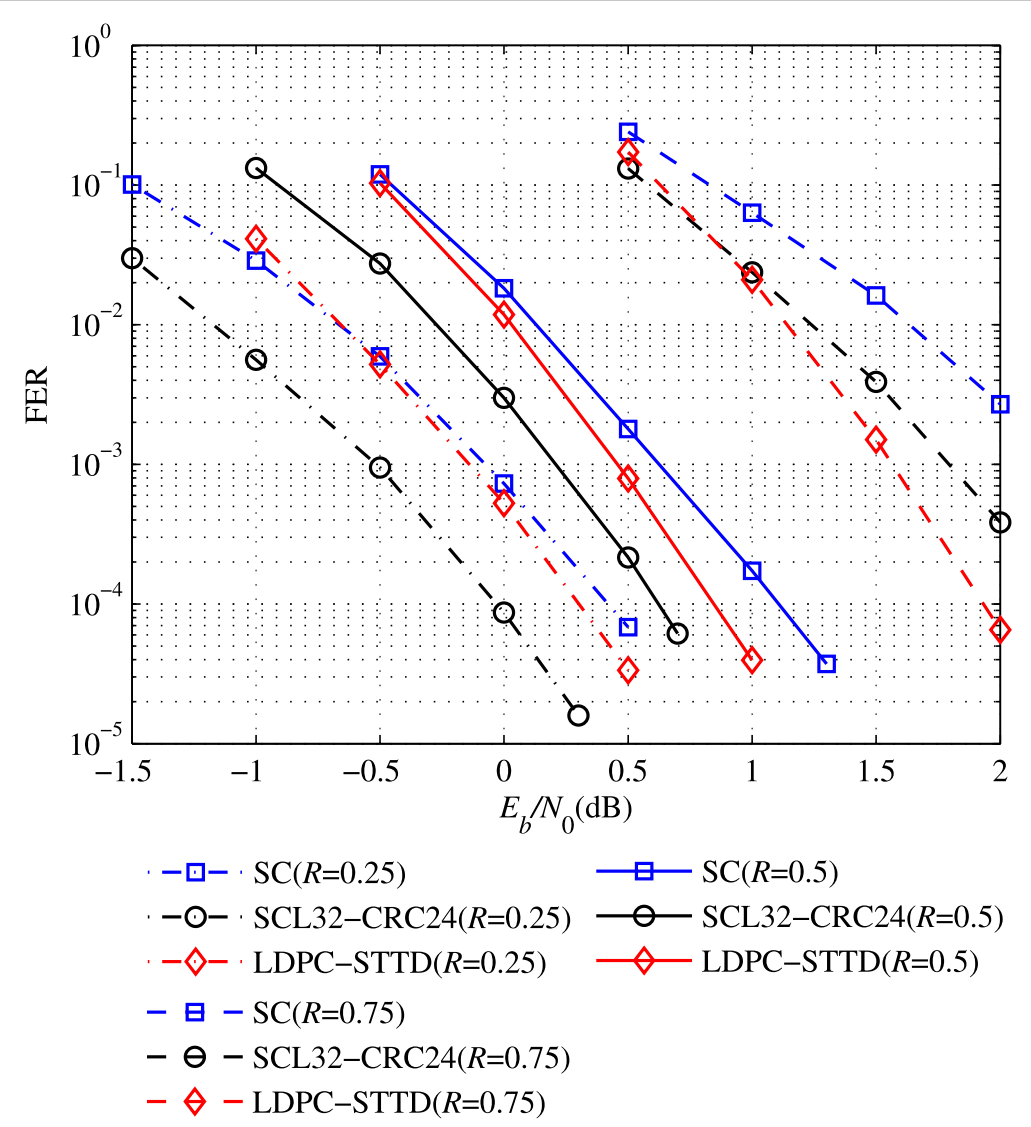

Fig. 6 FER performance of $2 \times 2$ polar-coded STTD system and LDPC-based STTD system. The frame error rate performance of polar-coded STTD system with two transmit antennas and two receive antennas, comparing with the LDPC-coded STTD system. SC decoding and SCL decoding are both adopted 


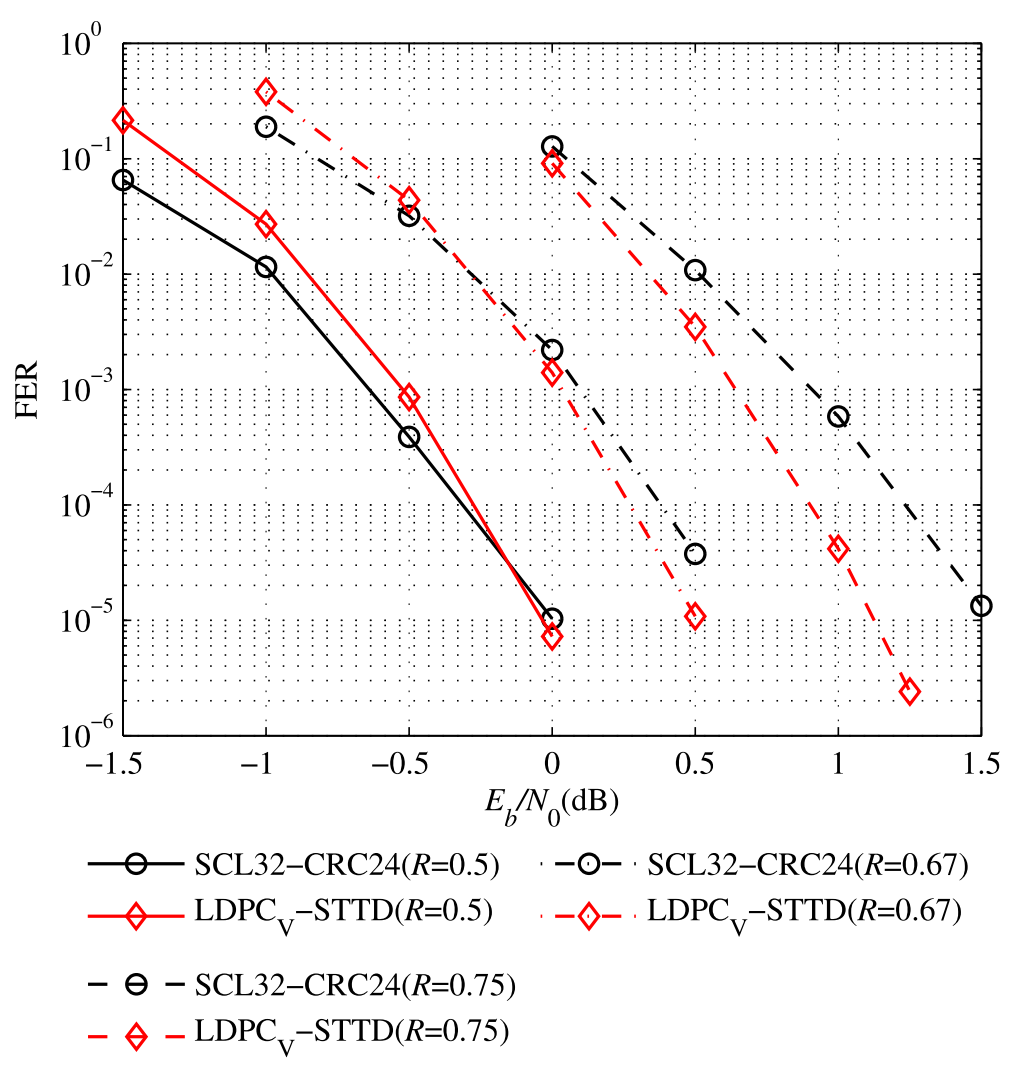

Fig. 7 FER performance of $2 \times 2$ polar-coded STTD system and state-of-the-art LDPC-based STTD system. The frame error rate performance of polar-coded STTD system with two transmit antennas and two receive antennas, comparing with the state-of-the-art LDPC-coded STTD system

polar codes is switched to 2048. According to the document, the length of the LDPC codes in the simulations is 1944 and the code rates $0.5,0.67$, and 0.75 are applied in the simulations. The corresponding averages of variable degree distributions are as $\bar{d}_{v}=3.583,3.667$, and 3.542 , respectively. The Rician $K$-factor of the channel is still assumed as $0 \mathrm{~dB}$. The CRC-aided SCL decoding is applied with the CRC parity bits 24 and list size $l=32$. At this point, the complexity of the SCL decoding is as $O(l N \log N)=O(720896)$. Then, we set the maximum BP iteration as $I_{\max }=50$ in order to balance the complexity of the BP decoding for LDPC codes with the one of the SCL decoding for polar codes approximatively. As shown in Fig. 7, the proposed polar-coded STTD system with the CRC-aided SCL decoding performs better than the state-of-the-art LDPC-based system when the code rate is $R=0.5$ and $E_{b} / N_{0}$ is not exceeding $-0.1 \mathrm{~dB}$. The state-of-the-art LDPC-based system provides better FER performance in waterfall region when the code rate increases to 0.67 and 0.75 . The polar code block becomes less reliable than LDPC block with rising the code rate, mainly because that more incompletely polarized subchannels are adopted to contain the information bits, which causes performance loss.

\section{Conclusions}

In this paper, a novel polar-coded STTD system is proposed to approach the capacity of MIMO. Density evolutions for our proposed scheme is analyzed in the Rician fading MIMO channels, which provide preferable guidance to construct polar codes. Simulation results show that as the number of antennas increases, the proposed systems will have better BER performance and less influence from the variable Rician $K$-factor of MIMO channels, simultaneously. Furthermore, we acknowledge that the $2 \times 2$ polar-coded STTD systems have a good tradeoff of cost and BER performance in practical applications. With the help of CRC-aided SCL decoding algorithm, the proposed polar-coded STTD system with $2 \times 2$ antennas can provide better FER performance than conventional LDPC-based system under low and mid code rates. The average advantage is $0.3 \mathrm{~dB}$ approximately when code rate is 0.25 and $0.2 \mathrm{~dB}$ when code rate is 0.5 .

\section{Abbreviations}

BER: Bit error rate; BP: Belief propagation; CDI: Channel distribution information; CRC: Cyclic redundancy check; CSI: Channel side information; DE: Density evolution; DMC: Discrete memoryless channel; FER: Frame error rate; LDPC: Low-density parity-check; LLR: Log-likelihood ratio; LOS: Direct line-of-sight; MIMO: Multiple-input multiple-output; PDF: Probability distribution function; 
STTD: Space-time transmit diversity; SC: Successive-cancellation; SCL: Successive-cancellation list; STC: Space-time coding

\section{Funding}

This work has been supported by the National Natural Sciences Foundation of China (NSFC) under grant 61771158, 61701136, 61525103, and 61371102; the National High Technology Research and Development Program no. 2014AA01A704; the Natural Scientific Research Innovation Foundation in Harbin Institute of Technology under grant HIT. NSRIF. 2017051; and the Shenzhen Basic Research Program under grant JCYJ20160328163327348 and JCYJ20150930150304185.

\section{Availability of data and materials}

The authors do not share the data, due to the requirements of the foundations.

\section{Authors' contributions}

$\mathrm{BF}$ and JJ conceived and designed the study. SG carried out most of the analyses. BF and SW performed the experiments. QZ reviewed and edited the manuscript. All authors read and approved the manuscript.

\section{Authors' information}

The authors are with the Communication Engineering Research Center, Harbin Institute of Technology (Shenzhen), Guangdong 518055, China.

\section{Competing interests}

The authors declare that they have no competing interests.

\section{Publisher's Note}

Springer Nature remains neutral with regard to jurisdictional claims in published maps and institutional affiliations.

Received: 4 July 2017 Accepted: 22 January 2018

Published online: 31 January 2018

\section{References}

1. E Arikan, Channel polarization: a method for constructing capacity-achieving codes for symmetric binary-input memoryless channels. IEEE Trans. Inf. Theory. 55(7), 3051-73 (2009)

2. S Iqbal, AA Hashmi, G Choi, Improved belief propagation decoding algorithm for short polar codes. Wirel. Pers. Commun. 96(1), 1437-49 (2017)

3. G Li, J Mu, X Jiao, J Guo, X Liu, Enhanced belief propagation decoding of polar codes by adapting the parity-check matrix. EURASIP J. Wirel. Commun. Netw. 2017(1), 40 (2017)

4. I Tal, A Vardy, List decoding of polar codes. IEEE Trans. Inf. Theory. 61(5), 2213-26 (2015)

5. G Sarkis, P Giard, A Vardy, C Thibeault, WJ Gross, Fast list decoders for polar codes. IEEE J. Sel. Areas Commun. 34(2), 318-28 (2016)

6. TKoike-Akino, Y Wang, SC Draper, K Sugihara, W Matsumoto, in Communications (ICC), 2017 IEEE International Conference On. Bit-interleaved polar-coded ofdm for low-latency $\mathrm{m} 2 \mathrm{~m}$ wireless communications (Paris, 2017), pp. 1-7

7. C-X Wang, F Haider, X Gao, X-H You, Y Yang, D Yuan, H Aggoune, H Haas, S Fletcher, E Hepsaydir, Cellular architecture and key technologies for $5 \mathrm{~g}$ wireless communication networks. IEEE Commun. Mag. 52(2), 122-30 (2014)

8. H Huang, CB Papadias, S Venkatesan, MIMO Communication for cellular networks. (Springer, New York, 2011)

9. MH Halmi, MY Alias, TC Chuah, Bandwidth efficient power-aided semi-blind channel estimation for multiple-antenna systems. IET Sig. Process. 7(7), 606-14 (2013)

10. SM Alamouti, A simple transmit diversity technique for wireless communications. IEEE J. Sel. Areas Commun. 16(8), 1451-8 (1998)

11. A Ohhashi, TOhtsuki, in Global Telecommunications Conference (GLOBECOM). Performance analysis and code design of low-density parity-check (LDPC) coded space-time transmit diversity (STTD) system (Dallas, 2004), pp. 3118-3122

12. K Wang, $\mathrm{H}$ Shen, W Wu, Z Ding, Joint detection and decoding in Idpc-based space-time coded mimo-ofdm systems via linear programming. IEEE Trans. Sig. Process. 63(13), 3411-24 (2015)
13. A Razi, M Ardebilipour, F Afghah, in 4th International Conference on Wireless Communications, Networking and Mobile Computing (WiCOM'08). Space-time block codes assisted by fast turbo codes, (Dalian, 2008), pp. 1-6

14. R Mori, T Tanaka, Performance of polar codes with the construction using density evolution. IEEE Commun. Lett. 13(7), 519-21 (2009)

15. I Tal, A Vardy, How to construct polar codes. IEEE Trans. Inf. Theory. 59(10), 6562-82 (2013)

16. B Feng, J Jiao, S Wang, S Wu, Q Zhang, in Vehicular Technology Conference (VTC-Fall), 2016 IEEE 84th. Construction of polar codes concatenated to space-time block coding in mimo system (Montreal, 2016), pp. 1-5

17. L Polak, T Kratochvil, Analysis and simulation of the transmission distortions of the mobile digital television dvb-sh, part 2: Satellite mode dvb-sh-b with tdm. Radioengineering. 21(1), 126-133 (2012)

18. GL Stuber, Principles of mobile communication vol. 2. (Kluwer Academic Publishers, Dutch, 2001)

19. K Niu, K Chen, J Lin, Q Zhang, Polar codes: primary concepts and practical decoding algorithms. IEEE Commun. Mag. 52(7), 192-203 (2014)

20. A Balatsoukas-Stimming, M Bastani Parizi, A Burg, Llr-based successive cancellation list decoding of polar codes. IEEE Trans. Signal Proc. 63(19), 5165-79 (2015)

21. TRichardson, R Urbanke, Mod. coding theory. (Cambridge University Press, Cambridge, 2008)

22. TJ Richardson, MA Shokrollahi, RL Urbanke, Design of capacity-approaching irregular low-density parity-check codes. IEEE Trans. Inf. Theory. 47(2), 619-37 (2001)

23. L Ma, Z Liang, D Liu, in 2017 International Conference on Cyber-Enabled Distributed Computing and Knowledge Discovery (CyberC). Performance of polarized channel coding in trpc-uwb communication systems, (Nanjing, 2017), pp. 466-470

24. S Jing, C Yang, J Yang, X You, C Zhang, in 20179 th International Conference on Wireless Communications and Signal Processing (WCSP). Joint detection and decoding of polar-coded scma systems, (Nanjing, 2017), pp. 1-6

25. J Dai, K Niu, Z Si, C Dong, J Lin, Does gaussian approximation work well for the long-length polar code construction? IEEE Access. 5, 7950-63 (2017)

26. DJ MacKay, RM Neal, Near shannon limit performance of low density parity check codes. Electron. Lett. 32(18), 1645 (1996)

27. Verizon, Verizon 5G TF; Air interface working group; Verizon 5th generation radio access; Multiplexing and channel coding (Release 1). TS V5G.212 V1.2, Jun. 2016 [EB/OL]. http://www.5gtf.org/V5G_212_v1p2.pdf

\section{Submit your manuscript to a SpringerOpen ${ }^{\circ}$ journal and benefit from:}

- Convenient online submission

- Rigorous peer review

- Open access: articles freely available online

- High visibility within the field

- Retaining the copyright to your article

Submit your next manuscript at $>$ springeropen.com 